\title{
DFT calculation of the Renner Teller Effect in NCO: Preliminary assessment of Exact Exchange Energy on the accuracy of the $\mathrm{X}^{2} \Pi$ Renner Coefficient
}

\author{
David Kashinski ${ }^{1}$, Tyler Radziewicz ${ }^{1}$, Matthew Suarez ${ }^{1}$, Constantine Stephens ${ }^{1}$, and \\ Edward Byrd ${ }^{2}$ \\ ${ }^{1}$ United States Military Academy at West Point \\ ${ }^{2}$ Army Research Laboratory Aberdeen Proving Ground
}

May 19, 2021

\begin{abstract}
Assessment of DFT methods through analysis of the Renner-Teller Effect (RTE) in the $\mathrm{X}^{2} \Pi$ state of the NCO radical was completed. Our results suggest that the amount of exact exchange at long range is important for an accurate description of the RTE in NCO. DFT functionals from the B3LYP, PBE, TPSS, M06, and M11 families with standard Correlation Consistent, 6-311G split valence family, as well as Sadlej, and Sapporo polarized triple- $\zeta$ basis sets were assessed. Our Renner coefficients are compared with previously published theoretical and experimental results to characterize the overall accuracy of various functional/basis set combinations in determining the RTE splitting in the $\Pi$ (bending) modes of $\mathrm{NCO}\left(\mathrm{X}^{2} \Pi\right)$. We suggest that this method of analysis can be extended to other systems, serve as an accuracy metric when selecting a functional, and provide a means to create training sets for machine learning in computational molecular physics applications.
\end{abstract}

\section{Hosted file}

main.pdf available at https://authorea.com/users/413467/articles/522561-dft-calculation-ofthe-renner-teller-effect-in-nco-preliminary-assessment-of-exact-exchange-energy-on-theaccuracy-of-the- $\mathrm{x} 2 \% \mathrm{CF} \% 80$-renner-coefficient 